\title{
ACE-INHIBITION AND PHYSICAL FUNCTION:
}

\author{
Results from the Trial of Angiotensin Converting Enzyme Inhibition and Novel \\ Cardiovascular Risk Factors (TRAIN) study
}

Matteo Cesari, MD, PhD ${ }^{1,2,3}$, Claudio Pedone, MD, PhD, MPH ${ }^{1,2}$, Raffaele Antonelli Incalzi, MD, PhD $^{1,2}$, and Marco Pahor, MD ${ }^{3}$

${ }^{1}$ Centro per la Salute dell'Anziano - Area di Geriatria, Università Campus Bio-Medico, Rome, Italy

2Fondazione "Alberto Sordi", Università Campus Bio-Medico, Rome, Italy

${ }^{3}$ Department of Aging and Geriatric Research, University of Florida - Institute on Aging, Gainesville, $\mathrm{FL}$

\section{Abstract}

Objectives-Aim of the present study is to evaluate whether an ACE inhibitor intervention is able to significantly improve physical performance and muscle strength in a sample of older persons.

Design-Double-blind, cross-over, randomized, placebo-controlled trial.

Setting-The Trail of Angiotensin Converting Enzyme Inhibition and Novel Cardiovascular Risk Factors (TRAIN) study.

Participants-Two hundred fiftyseven subjects aged 55 years and older with high cardiovascular risk profile.

Intervention-Six month of fosinopril use versus placebo.

Measurements-The Short Physical Performance Battery score (rescaled to obtain a continuous variable ranging from 0 to 3 points), and the hand grip strength were measured at the baseline visit, and after 6 and 12 months of follow-up. Paired t-test analyses were performed to compare results of physical function measures after ACE inhibition and placebo interventions.

Results-Mean age of the sample population was 65.97 (standard deviation 7.41) years old. No statistically significant difference was found at the Short Physical Performance Battery $(\mathrm{p}=0.23)$ and hand grip strength $(\mathrm{p}=0.57)$ results after ACE inhibition (2.113, standard deviation [SD] 0.284; and $37.044 \mathrm{~kg}$, SD $12.993 \mathrm{~kg}$, respectively) compared to placebo (2.096, SD 0.298 ; and $36.898 \mathrm{~kg}$, SD $13.178 \mathrm{~kg}$, respectively). No significant effects from ACE inhibition were also found when the three subtests composing the Short Physical Performance Battery (i.e., 4-meter walking speed, balance, and chair stand tests) were separately analyzed. Consistent negative results were obtained after analyses were restricted to participants showing the highest compliance to treatment and/or receiving the maximum fosinopril dosage.

(C) 2009 American Medical Directors Association. Published by Elsevier Inc. All rights reserved.

Corresponding Author: Matteo Cesari, MD, PhD. Centro per la Salute dell' Anziano - Area di Geriatria, Università Campus BioMedico, Via Alvaro del Portillo 5, 00128 Rome, Italy; Phone: +39 (06) 2254-1654.; Fax: +39 (06) 2254-1602; macesari@ gmail.com. None of the Authors had significant conflict of interest to disclose for the present work.

Publisher's Disclaimer: This is a PDF file of an unedited manuscript that has been accepted for publication. As a service to our customers we are providing this early version of the manuscript. The manuscript will undergo copyediting, typesetting, and review of the resulting proof before it is published in its final citable form. Please note that during the production process errors may be discovered which could affect the content, and all legal disclaimers that apply to the journal pertain. 
Conclusion-No significant modifications in physical performance and muscle strength were reported after 6 months of fosinopril use in older persons with high cardiovascular risk profile. Given these negative findings, it is possible that the beneficial effects of ACE inhibitors on physical function might be due to the activation of a virtuous cycle determined by an improved cardiovascular system. Further specifically designed studies are needed to confirm our findings, and expand them to different populations and ACE inhibitors. If our findings will be confirmed, the extra-cardiovascular properties of ACE inhibitors in older persons might be substantially resized.

\section{Keywords}

Fosinopril; Physical function; ACE inhibition; Muscle strength

\section{INTRODUCTION}

Aging has been clearly associated with physical performance and muscle strength decline, potentially leading to disability in later life. Loss of physical function represents a major public health issue, especially considering the growing number of older persons in Western Countries1. Several preventive measures have been proposed as potentially able to halt (or, at least, delay) the onset of the disabling process. Unfortunately, the only intervention showing the most promising results is physical exercise ${ }^{2}$. To date, there are no definitive pharmacological interventions proven to prevent physical function decline. The only exception may be represented by angiotensin-converting enzyme (ACE) inhibitors3.

ACE inhibitors have not only been associated with major clinical benefits (e.g., reduction of morbidity, hospitalizations, and mortality) in patients with cardiovascular and metabolic conditions ${ }^{4-8}$. Several studies, both in animal3, 9 and human $10^{-12}$ models, have suggested that ACE inhibitors may provide significant benefits on physical function in older persons. It has been proposed that ACE inhibitors may improve physical function through a direct beneficial effect on skeletal muscle and by promoting virtuous metabolic pathways (e.g., improvement of body composition, reduction of inflammatory status, reduction of insulin resistance...)

13-16. Unfortunately, this matter is still largely debated, and studies confuting this relationship are available as well17-19. A major limit of current evidence, concurring in feeding the existent controversy, is the limited number of clinical trials specifically exploring this topic ${ }^{12}, 20-22$. Further studies confirming and expanding current evidence have been encouraged ${ }^{23}$.

Aim of the present study is to evaluate whether an ACE inhibitor intervention (i.e., fosinopril) is able to significantly improve physical performance and muscle strength in a sample of older persons with high cardiovascular risk profile. The present analyses were conducted using data from the Trial of Angiotensin Converiting Enzyme Inhibition and Novel Cardiovascular Risk Factors (TRAIN) study $24,25$.

\section{METHODS}

The TRAIN study

The present analyses were conducted using data from participants enrolled in the TRAIN study 24,25 . The TRAIN study design and methods have been previously described. Briefly, the TRAIN study is a double-blind, cross-over, randomized, placebo-controlled trial aimed at assessing the biological mechanisms by which ACE-inhibition (i.e., fosinopril) may improve clinical outcomes in persons aged 55 years or older with a high cardiovascular risk profile. In a cross-over design each participant is randomized to a sequence of treatments. Advantages of this design include the ability to control for all known and unknown confounding variables with implicit matching of data, improved recruitment (because every participant receives the 
intervention), and a substantially higher statistical power compared to the independent parallel groups design.

To be included in the TRAIN study, participants had to present with at least one of the following indicators of high cardiovascular risk: 1) coronary heart disease; 2) peripheral vascular disease; 3) history of stroke ( $>6$ months); 4) diabetes with at least one other cardiovascular risk factor (hypertension, total cholesterol $>200 \mathrm{mg} / \mathrm{dl}$, HDL cholesterol $<40 \mathrm{mg} / \mathrm{dl}$ for men or $<50 \mathrm{mg} /$ dl for women, triglycerides $\geq 150 \mathrm{mg} / \mathrm{dl}$, current cigarette smoking, obesity [body mass index, $\mathrm{BMI} \geq 30 \mathrm{~kg} / \mathrm{m}^{2}$ or waist $>102 \mathrm{~cm}$ for men and $>88 \mathrm{~cm}$ for women], known microalbuminuria, any evidence of previous vascular disease). Exclusion criteria for the TRAIN study were: 1 ) current use of or known hypersensitivity to ACE inhibitors; 2) diagnosis of specific cardiovascular conditions (including previous myocardial infarction, ejection fraction $<40 \%$, hemodynamically significant primary valvular or outflow tract obstruction, constrictive pericarditis, complex congenital heart disease, syncopal episodes likely to be due to lifethreatening arrhythmias, uncontrolled hypertension, cor pulmonale, planned cardiac surgery or angioplasty within three months); 3) conditions affecting results of or safe participation in the trial (significant renal disease [i.e. renal artery stenosis; creatine clearance $<0.6 \mathrm{~mL} / \mathrm{s}$ or serum creatinine $\geq 2.26 \mathrm{mg} / \mathrm{dL}$; overt nephropathy; serum potassium $>5.5 \mathrm{mEq} / \mathrm{L}]$, lifethreatening illness, recent surgical procedure ( $<6$ months), simultaneous enrollment in another experimental drug trial); 4) compliance issues (plans to leave the area in the next three months, substance abuse, compliance $<80 \%$ during the pre-randomization phase). All the inclusion and exclusion criteria were based on medical history, medical records review, physical examination, and laboratory data of participants.

All the participants signed an informed consent for the study at the screening visit. The Wake Forest University School of Medicine Institutional Review Board approved the study protocol.

\section{Study participants}

Participants were recruited from the communities of Winston Salem, NC and Greensboro, NC. After a pre-screening phone interview (phone contacts=2,347), a clinical visit aimed at reviewing medical history and records of potential participants was arranged for those $(n=576)$ who successfully met the study inclusion and exclusion criteria. Subjects who successfully completed this pre-screening visit $(n=401)$ were entered in a screening and single-blind oneweek run-in phase, in which the tolerability of ACE-inhibitor and compliance were evaluated.

Participants who successfully completed all the preliminary phase interviews and visits $(n=294)$ were then randomized to placebo or active intervention (i.e., treatment with fosinopril) of the study during the baseline clinical visit. After 3 visits in a 6-month follow-up, the crossover occurred. After further 6 months and 3 mid-term clinical visits, participants attended closeout visits. A special safety visit was also performed one week after the cross-over. The drug titration and follow-up procedures of the first phase were repeated in the following 6 months of the trial.

The present analyses are conducted on a sample of 257 participants, after exclusion of 37 participants for which none of the physical function measures of interest was assessed. Participants with the physical function measurements at all time points (baseline, 6-month, and 12-month clinic visits) were 222 for the Short Physical Performance Battery, 225 for the hand grip strength, 225 for the 4-meter walking speed. No significant sociodemographic, clinical, and biological differences were present between participants considered in the present analyses and those excluded because of missing values. 


\section{Intervention}

At randomization, patients were assigned to receive fosinopril versus matching placebo at a dose of $20 \mathrm{mg}$ daily for one week, then $40 \mathrm{mg}$ daily. If the dosage was not tolerated because of hypotension, cough or other problems, the participants were given the lowest tolerated dose. The rationale for use of high dose fosinopril was the attempt to maximize potential effects on the biomarkers of interest, despite minimal differences in blood pressure control seen with higher doses (about 2-3 mmHg)26, 27. Fosinopril was chosen over other ACE-inhibitos because of the more favorable pharmacokinetic profile (i.e. high trough-to-peak ratio, both renal and hepatic elimination)28, 29, low incidence of adverse events 30,31 , and overall evidence suggesting comparable efficacy of different ACE-inhibitors32 $2^{-34}$. The active form half-life is about 12 hours. Fosinopril was donated by Bristol-Myers Squibb (New York, NY).

Study drug was supplied at an interim 3-month visit. Before each visit, investigators assessed the participant's adherence by counting the study pills left. In a previous study, the efficacy of the conducted intervention in terms of angiotensin-II and blood pressure reductions has already been documented in the TRAIN study ${ }^{24}$.

\section{Physical performance and muscle strength measures}

Short Physical Performance Battery-Performance of the lower extremities was assessed using the Short Physical Performance Battery, composed by three subtests, that are the walking speed, chair-stand, and balance tests. Walking speed was defined as the best performance (time in seconds) of two 4-meter walks at a usual pace along a corridor. For the chair-stand test, the participant was asked to rise from a chair and sit down 5 times as quickly as possible with hands folded across the chest. The performance was expressed as total time (in seconds) to complete the test. For the standing balance test, participants were asked to stand in three increasingly challenging positions for 10 seconds each: a side-by-side-feet standing position, a semitandem position, and a full tandem position.

Arithmetic performance scores were calculated to obtain continuous measures35, ${ }^{36}$. The timed scores of the performance tests were rescaled to values ranging from 0 (worst performance) to 1 (best performance). Worst performers were participants who were unable to complete the task or who had a performance above the worst $99^{\text {th }}$ gender-specific percentile. The following formulas were applied to rescale the measures: Walking speed: $1-[(0.52 \mathrm{~m} / \mathrm{sec}$ for men or 0.40 $\mathrm{m} / \mathrm{sec}$ for women) divided for speed in $\mathrm{m} / \mathrm{sec}$ ]; Chair-stand test: 1-[time in seconds divided for 26.76 seconds (men) or 30.18 seconds (women)]; Standing balance test: time in seconds divided for 30 seconds (for both men and women).

A summary physical performance score ranging from 0 to 3 was calculated by adding these three rescaled scores. This approach, resulting in continuous scores of the Short Physical Performance Battery, has been previously adopted ${ }^{35,36}$. By using continuous variables instead of scores based on the "traditional" categorization of the Short Physical Performance Battery according to pre-defined cut-points, we have increased the capacity of our analyses to capture even minimal changes in physical performance tests results.

Hand grip strength-The hand grip strength test was measured using a hand-held dynamometer (Jamar hydraulic hand dynamometer, Fred Sammons Inc., Burr Ridge, IL). Participants were asked to perform the task twice with each hand. The best result was used for the present analyses.

\section{Sample population characteristics}

Sociodemographic (age, gender, race, current smoking) and self-reported clinical conditions (angina, cancer, diabetes, hypertension, pulmonary disease, myocardial infarction, peripheral 
artery disease, stroke, gastric ulcer) were collected at the baseline assessment. BMI was calculated as body mass (in kilograms) divided by squared height (in meter ${ }^{2}$ ). Body mass was measured by Delphi (Hologic Inc., Bedford, MA) dual-energy X-ray absorptiometry (DXA) scan. Height was self-reported by participants.

\section{Statistical analysis}

The mean values of physical function measures were calculated for mid-term and end of followup visits (visits 6 and 12, respectively). The effect of fosinopril was evaluated on results obtained from the assessment of physical function measures performed at the 6-month visit for participants receiving the medication during the first part of the trial, and at the 12-month visit for participants receiving fosinopril in the second part of the trial. Similarly, the effect of placebo was determined at the 6-month visit for participants receiving placebo during the first part of the trial, and at the 12-month visit for those receiving placebo during the second part of the trial. Paired t-test analyses were performed to compare results of physical function tests between fosinopril and placebo interventions. Because all comparisons are done by matched pairs, no adjustment is needed for baseline factors, such as age, gender, or other potential confounders. A value of $\mathrm{p}<0.05$ was considered as statistically significant. Analyses were performed using SPSS version 16.0 software (SPSS Inc., Chicago, IL).

\section{RESULTS}

Main characteristics of the study participants $(n=257)$ are presented in Table 1 . The study sample had a mean age of 65.97 (standard deviation, SD 7.41) years, with men (57.6\%) and Whites $(74.3 \%)$ more prevalent compared to women (42.4\%) and other races (25.7), respectively. Given the inclusion criteria of the TRAIN study, the sample population was characterized by a high cardiovascular risk profile. In fact, participants tended to be overweight (mean BMI 28.98 [SD 4.73] kg/m²), and report a high prevalence of hypertension $(60.7 \%$ ), diabetes $(23.3 \%)$, coronary heart disease $(12.5 \%)$, and history of stroke (8.9\%). Overall, the study sample showed a good physical function. In fact, mean results at the hand grip strength, Short Physical Performance Battery score, and 4-meter walking speed of participants were 39.02 (SD 13.76) kg, 2.088 (SD 0.242) points [corresponding to 10.37 (SD 1.43) points at the traditional scoring], and 0.611 (SD 0.106) points [corresponding to 1.25 (SD 0.25) $\mathrm{m} / \mathrm{sec}$ ], respectively. No significant differences (all p values $>0.1$ ) were present between participants randomized to placebo vs. those randomized to fosinopril during the first phase of the trail.

In Table 2, results from t-test analyses comparing results of physical function measures obtained at the end of the study interventions (i.e., fosinopril vs. placebo) are reported. No statistically significant differences for both the Short Physical Performance Battery (fosinopril: 2.113 [SD 0.284]; placebo: 2.096 [SD 0.298]; $\mathrm{p}=0.23$ ) and the hand grip strength (fosinopril: 37.044 [SD 12.993] kg; placebo: 36.898 [SD 13.178] kg; $\mathrm{p}=0.57$ ) were reported between the fosinopril and placebo interventions. No statistically significant effect was also reported when the three subtests composing the Short Physical Performance Battery (i.e., walking speed, balance, and chair stand tests) were separately analyzed (all $\mathrm{p}$ values $>0.2$ ).

Consistent negative results were also found after analyses were restricted to participants showing high compliance (i.e., higher than $70 \%$ or $80 \%$ ) to the treatment and/or receiving the maximum dosage of fosinopril (i.e. $40 \mathrm{mg}$ daily). Similarly, non-statistically significant results were obtained if analyses were performed considering the Short Physical Performance Battery as a 13-level categorical variable (as described by Guralnik and colleagues37, 38), and/or the 4-meter walking speed in $\mathrm{m} / \mathrm{sec}$.

In the above-described analyses, physical function results obtained after ACE inhibition were compared to those measured at the end of the 6-month placebo administration (i.e. 6-month 
clinic visit for participants randomized to placebo during the first phase of the trial, and 12month clinic visit for participants randomized to placebo during the second phase of the trial). Similar non-statistically significant findings were also reported if results obtained from the assessments of physical function performed at baseline clinic visit were considered as placebo values.

\section{DISCUSSION}

Our results show no statistically significant improvements in a well-established measure of physical performance (i.e., the Short Physical Performance Battery) and a commonly adopted marker of muscle strength (i.e., hand grip strength) after a 6-month fosinopril intervention. These negative findings were not substantially modified when analyses were restricted to the most compliant participants and/or those receiving the maximum ACE inhibitor dosage.

During the last decade, ACE inhibitors have growingly been indicated as potentially able to prevent and/or delay the disabling process. This hypothesis is mainly funded on a large body of evidence (mostly from observational studies) suggesting ACE inhibition as able to reduce the inflammatory status and improve body composition. To our knowledge, very few clinical trials have specifically explored the effects of an ACE inhibitor intervention on measures of physical function.

Recently, Sumukadas and colleagues ${ }^{12}$ reported positive findings from a double-blind randomized clinical trial evaluating the effect of a perindopril intervention on physical function in 130 older persons (aged 65 years and older) with self-reported mobility or functional impairment. In particular, Authors tested whether a 20-week perindopril intervention was able to improve results at the 6-minute walking test (primary outcome of the study). Participants in the perindopril intervention group reported a significant mean improvement (about 31.4 meters) in the 6-minute walking test compared to participants in the placebo group at the end of the study. Similar findings had previously been reported from analyses conducted by Cleland and colleagues ${ }^{20}$ and by Hutcheon and colleagues 21 in older persons with heart failure.

Differently from these previous studies, our analyses failed to report significant improvements in physical performance and muscle strength following the ACE inhibitor intervention. Although a class effect is usually considered for ACE inhibitors ${ }^{32-} 34$, it is noteworthy that studies reporting positive findings used perindopril12, 20, 21, while different ACE inhibitors did not significantly affect physical function in our study (adopting fosinopril as study drug) and in the $\mathrm{Zi}$ and colleagues' study ${ }^{22}$ (using quinapril).

Other possible explanations can also be provided to explain the different results obtained by these trials. For example, it is noteworthy that, among the secondary analyses performed by Sumukadas and colleagues ${ }^{12}$, negative results (consistently with ours) were reported for the sit-to-stand test (similar to the chair stand test included in the Short Physical Performance Battery and used in this study). It is possible that the reported positive results were specifically due to some specific characteristics of the 6-minute walking test. Shorter length walking tests and muscle strength measures (as those considered in our study, or the sit-to-stand test) may differently stress those organism subsystems required for functioning.

The different characteristics of the study samples should also be noted. In our study, we recruited participants with a high risk cardiovascular profile due to the need to maximize those biological pathways (e.g., inflammation, endothelial dysfunction, hemostatis modifications, ...) associated with the atherosclerotic disease and potentially influenced by the ACE inhibition (as the TRAIN primary objective required). Sumukadas and colleagues ${ }^{12}$ performed their study in an older sample population (mean age 78.7 years old) characterized by mobility and/or functional limitation. Other studies were specifically designed in patients with heart 
failure20-22. It might be that the better physical function showed by our participants might have determined a "ceiling effect" in the reported results.

Despite our negative findings, we cannot ignore the growing body of evidence proposing an important role of ACE inhibitors in the maintenance of physical function at older age. The nature of these positive effects has not yet been biologically clarified. However, the cardiovascular protection properties of ACE inhibitors are well-established in literature 5,10 , 39,40 . The role played by a healthy cardiovascular status on successful aging should not be underestimated ${ }^{41}, 42$. In fact, the beneficial effects of ACE inhibitors on physical performance might be simply due to the activation of a virtuous cycle determined by an improved cardiovascular system.

Several limitations of our study should be acknowledged. First of all, it is possible that our analyses had not the adequate statistical power to exclude the risk of type II errors. We have estimated that our sample size was sufficient to detect true differences (with statistical power $=0.80$, and $\alpha=0.05$ ) of \pm 0.039 points, $\pm 0.035 \mathrm{~m} / \mathrm{sec}$, and $\pm 0.716 \mathrm{~kg}$ at the Short Physical Performance Battery total score (ranging from 0 to 3 ), the 4-meter walking speed, and the hand grip strength, respectively. Moreover, to date, this is one of the largest randomized clinical trial with longer follow-up on this topic. As previously mentioned, the characteristics of our sample population as well as the adopted ACE inhibitor (i.e., fosinopril) may limit the generalizability of our findings. It is also possible that the detection of significant modifications in physical function measures following ACE inhibition may need a longer exposure to be captured. Before clinical changes might be evident, relevant and prolonged improvements of the pathophysiological pathways responsible for the disabling cascade may be required. However, in this context, it is important to take into account the negative effects of ACE inhibition on biomarkers of inflammation we previously reported ${ }^{24}$. Finally, the cross-over design of the TRAIN study might have influenced our findings if the intervention during the first period cured the studied condition (so that the participant could not return to the initial state during the wash-out phase). In other words, our analyses imply that the study drug had no carry-over effect on the outcomes after the participant was taken off ACE inhibition.

\section{CONCLUSIONS}

Our data show no significant modifications in physical performance and muscle strength after 6-month of fosinopril use in older persons with high cardiovascular risk profile. If our findings will be confirmed, the great enthusiasm about the extra-cardiovascular properties of ACE inhibitors in older persons might be substantially resized. Further specifically designed studies are needed to confirm our findings, and expand them to different populations and ACE inhibitors.

\section{Acknowledgments}

The TRAIN study is National Institute of Health-funded project (NIH grant R01-HL68901). The TRAIN study was also (partially) supported by the University of Florida Claude D. Pepper Older Americans Independence Center (NIH grant 1P30-AG028740), Wake Forest University Claude D. Pepper Older Americans Independence Center (NIH grant 5P30-AG021332), and the Wake Forest University General Clinical Research Center (NIH grant M01-RR07122). Fosinopril was donated by Bristol-Myers Squibb (New York, NY).

\section{REFERENCES}

1. Guralnik JM, Fried LP, Salive ME. Disability as a public health outcome in the aging population. Annu Rev Public Health 1996;17:25-46. [PubMed: 8724214] 
2. Pahor M, Blair SN, Espeland M, et al. Effects of a physical activity intervention on measures of physical performance: Results of the lifestyle interventions and independence for Elders Pilot (LIFE-P) study. J Gerontol A Biol Sci Med Sci 2006;61:1157-1165. [PubMed: 17167156]

3. Carter CS, Onder G, Kritchevsky SB, Pahor M. Angiotensin-converting enzyme inhibition intervention in elderly persons: effects on body composition and physical performance. J Gerontol A Biol Sci Med Sci 2005;60:1437-1446. [PubMed: 16339331]

4. Effect of enalapril on mortality and the development of heart failure in asymptomatic patients with reduced left ventricular ejection fractions. The SOLVD Investigattors. N Engl J Med 1992;327:685691. [PubMed: 1463530]

5. Efficacy of atenolol and captopril in reducing risk of macrovascular and microvascular complications in type 2 diabetes: UKPDS 39. UK Prospective Diabetes Study Group. BMJ 1998;317:713-720. [PubMed: 9732338]

6. Estacio RO, Jeffers BW, Hiatt WR, Biggerstaff SL, Gifford N, Schrier RW. The effect of nisoldipine as compared with enalapril on cardiovascular outcomes in patients with non-insulin-dependent diabetes and hypertension. N Engl J Med 1998;338:645-652. [PubMed: 9486993]

7. Tatti P, Pahor M, Byington RP, et al. Outcome results of the Fosinopril Versus Amlodipine Cardiovascular Events Randomized Trial (FACET) in patients with hypertension and NIDDM. Diabetes Care 1998;21:597-603. [PubMed: 9571349]

8. Yusuf S, Sleight P, Pogue J, Bosch J, Davies R, Dagenais G. Effects of an angiotensin-convertingenzyme inhibitor, ramipril, on cardiovascular events in high-risk patients. The Heart Outcomes Prevention Evaluation Study Investigators. N Engl J Med 2000;342:145-153. [PubMed: 10639539]

9. Coirault C, Langeron O, Lambert F, et al. Impaired skeletal muscle performance in the early stage of cardiac pressure overload in rabbits: beneficial effects of angiotensin-converting enzyme inhibition. J Pharmacol Exp Ther 1999;291:70-75. [PubMed: 10490888]

10. Coirault C, Hagege A, Chemla D, Fratacci MD, Guerot C, Lecarpentier Y. Angiotensin-converting enzyme inhibitor therapy improves respiratory muscle strength in patients with heart failure. Chest 2001;119:1755-1760. [PubMed: 11399702]

11. Onder G, Penninx BW, Balkrishnan R, et al. Relation between use of angiotensin-converting enzyme inhibitors and muscle strength and physical function in older women: an observational study. Lancet 2002;359:926-930. [PubMed: 11918911]

12. Sumukadas D, Witham MD, Struthers AD, McMurdo ME. Effect of perindopril on physical function in elderly people with functional impairment: a randomized controlled trial. CMAJ 2007;177:867874. [PubMed: 17923654]

13. Carter CS, Cesari M, Ambrosius WT, et al. Angiotensin-converting enzyme inhibition, body composition, and physical performance in aged rats. J Gerontol A Biol Sci Med Sci 2004;59:416423. [PubMed: 15123750]

14. Sumukadas D, Struthers AD, McMurdo ME. Sarcopenia--a potential target for Angiotensinconverting enzyme inhibition? Gerontology 2006;52:237-242. [PubMed: 16849867]

15. Bauer JM, Kaiser MJ, Sieber CC. Sarcopenia in nursing home residents. J Am Med Dir Assoc 2008;9:545-551. [PubMed: 19083287]

16. Yeh SS, Lovitt S, Schuster MW. Pharmacological treatment of geriatric cachexia: evidence and safety in perspective. J Am Med Dir Assoc 2007;8:363-377. [PubMed: 17619035]

17. Bahi L, Koulmann N, Sanchez H, et al. Does ACE inhibition enhance endurance performance and muscle energy metabolism in rats? J Appl Physiol 2004;96:59-64. [PubMed: 12949022]

18. Gray SL, LaCroix AZ, Aragaki AK, et al. Angiotensin-converting enzyme inhibitor use and incident frailty in women aged 65 and older: prospective findings from the Women's Health Initiative Observational Study. J Am Geriatr Soc 2009;57:297-303. [PubMed: 19207145]

19. Schellenbaum GD, Smith NL, Heckbert SR, et al. Weight loss, muscle strength, and angiotensinconverting enzyme inhibitors in older adults with congestive heart failure or hypertension. J Am Geriatr Soc 2005;53:1996-2000. [PubMed: 16274385]

20. Cleland JG, Tendera M, Adamus J, Freemantle N, Polonski L, Taylor J. The perindopril in elderly people with chronic heart failure (PEP-CHF) study. Eur Heart J 2006;27:2338-2345. [PubMed: 16963472] 
21. Hutcheon SD, Gillespie ND, Crombie IK, Struthers AD, McMurdo ME. Perindopril improves six minute walking distance in older patients with left ventricular systolic dysfunction: a randomised double blind placebo controlled trial. Heart 2002;88:373-377. [PubMed: 12231595]

22. Zi M, Carmichael N, Lye M. The effect of quinapril on functional status of elderly patients with diastolic heart failure. Cardiovasc Drugs Ther 2003;17:133-139. [PubMed: 12975595]

23. Cranney A. Is there a new role for angiotensin-converting-enzyme inhibitors in elderly patients? CMAJ 2007;177:891-892. [PubMed: 17923657]

24. Cesari M, Kritchevsky SB, Atkinson HH, et al. Angiotensin-converting enzyme inhibition and novel cardiovascular risk biomarkers: results from the Trial of Angiotensin Converting Enzyme Inhibition and Novel Cardiovascular Risk Factors (TRAIN) study. Am Heart J 2009;157:334.e1-334.e8. [PubMed: 19185642]

25. Cesari M, Kritchevsky SB, Baumgartner RN, et al. Sarcopenia, obesity, and inflammation--results from the Trial of Angiotensin Converting Enzyme Inhibition and Novel Cardiovascular Risk Factors study. Am J Clin Nutr 2005;82:428-434. [PubMed: 16087989]

26. Pahor M, Franse LV, Deitcher SR, et al. Fosinopril versus amlodipine comparative treatments study: a randomized trial to assess effects on plasminogen activator inhibitor-1. Circulation 2002;105:457461. [PubMed: 11815428]

27. Gullestad L, Aukrust P, Ueland T, et al. Effect of high- versus low-dose angiotensin converting enzyme inhibition on cytokine levels in chronic heart failure. J Am Coll Cardiol 1999;34:2061-2067. [PubMed: 10588224]

28. Greenbaum R, Zucchelli P, Caspi A, et al. Comparison of the pharmacokinetics of fosinoprilat with enalaprilat and lisinopril in patients with congestive heart failure and chronic renal insufficiency. $\mathrm{Br}$ J Clin Pharmacol 2000;49:23-31. [PubMed: 10606834]

29. Piepho RW. Overview of the angiotensin-converting-enzyme inhibitors. Am J Health Syst Pharm 2000;57(Suppl 1):S3-7. [PubMed: 11030016]

30. Hansson L, Forslund T, Hoglund C, et al. Fosinopril versus enalapril in the treatment of hypertension: a double-blind study in 195 patients. J Cardiovasc Pharmacol 1996;28:1-5. [PubMed: 8797128]

31. Wagstaff AJ, Davis R, McTavish D. Fosinopril: a reappraisal of its pharmacology and therapeutic efficacy in essential hypertension. Drugs 1996;51:777-791. [PubMed: 8861547]

32. Garg R, Yusuf S. Overview of randomized trials of angiotensin-converting enzyme inhibitors on mortality and morbidity in patients with heart failure. Collaborative Group on ACE Inhibitor Trials. JAMA 1995;273:1450-1456. [PubMed: 7654275]

33. Neal B, MacMahon S, Chapman N. Effects of ACE inhibitors, calcium antagonists, and other bloodpressure-lowering drugs: results of prospectively designed overviews of randomised trials. Blood Pressure Lowering Treatment Trialists' Collaboration. Lancet 2000;356:1955-1964. [PubMed: 11130523]

34. Pahor M, Psaty BM, Alderman MH, et al. Health outcomes associated with calcium antagonists compared with other first-line antihypertensive therapies: a meta-analysis of randomised controlled trials. Lancet 2000;356:1949-1954. [PubMed: 11130522]

35. Onder G, Penninx BW, Lapuerta P, et al. Change in physical performance over time in older women: the Women's Health and Aging Study. J Gerontol A Biol Sci Med Sci 2002;57:M289-93. [PubMed: 11983722]

36. Cesari M, Penninx BW, Pahor M, et al. Inflammatory markers and physical performance in older persons: the InCHIANTI study. J Gerontol A Biol Sci Med Sci 2004;59:242-248. [PubMed: 15031308]

37. Guralnik JM, Ferrucci L, Pieper CF, et al. Lower extremity function and subsequent disability: consistency across studies, predictive models, and value of gait speed alone compared with the short physical performance battery. J Gerontol A Biol Sci Med Sci 2000;55:M221-31. [PubMed: 10811152]

38. Guralnik JM, Ferrucci L, Simonsick EM, Salive ME, Wallace RB. Lower-extremity function in persons over the age of 70 years as a predictor of subsequent disability. N Engl J Med 1995;332:556561. [PubMed: 7838189]

39. Ferrari R. Effects of angiotensin-converting enzyme inhibition with perindopril on left ventricular remodeling and clinical outcome: results of the randomized Perindopril and Remodeling in Elderly 
with Acute Myocardial Infarction (PREAMI) Study. Arch Intern Med 2006;166:659-666. [PubMed: 16567606]

40. Konstam MA, Rousseau MF, Kronenberg MW, et al. Effects of the angiotensin converting enzyme inhibitor enalapril on the long-term progression of left ventricular dysfunction in patients with heart failure. SOLVD Investigators. Circulation 1992;86:431-438. [PubMed: 1638712]

41. Burke GL, Arnold AM, Bild DE, et al. Factors associated with healthy aging: the cardiovascular health study. J Am Geriatr Soc 2001;49:254-262. [PubMed: 11300235]

42. Newman AB, Arnold AM, Naydeck BL, et al. "Successful aging": effect of subclinical cardiovascular disease. Arch Intern Med 2003;163:2315-2322. [PubMed: 14581251] 


\section{TABLE 1}

Main characteristics of the study participants $(n=257)$.

\begin{tabular}{|c|c|c|c|}
\hline & $\begin{array}{c}\underset{\mathbf{O}}{\text { Overall sample }} \mathbf{2 5 7} \\
\mathrm{n}\end{array}$ & $\begin{array}{c}\text { Placebo } \rightarrow \text { Fosinopril } \\
\mathbf{n}=127\end{array}$ & $\underset{n=130}{\text { Fosinopril }} \rightarrow$ Placebo \\
\hline Age (years) & $65.97 \pm 7.41$ & $65.94 \pm 7.23$ & $66.0 \pm 7.60$ \\
\hline Gender (women) & 42.4 & 44.1 & 40.8 \\
\hline \multicolumn{4}{|l|}{ Race } \\
\hline * White & 74.3 & 74.8 & 73.8 \\
\hline * Black & 23.7 & 23.6 & 23.8 \\
\hline$*$ Other & 2.0 & 1.6 & 2.6 \\
\hline Body Mass Index $\left(\mathrm{kg} / \mathrm{m}^{2}\right)$ & $28.98 \pm 4.73$ & $28.90 \pm 4.68$ & $29.05 \pm 4.79$ \\
\hline Current smoking & 11.7 & 9.4 & 13.8 \\
\hline Coronary heart disease & 12.5 & 13.4 & 11.5 \\
\hline Cancer & 14.4 & 13.4 & 15.4 \\
\hline Diabetes & 23.3 & 26.8 & 20.0 \\
\hline Hypertension & 60.7 & 65.4 & 56.2 \\
\hline Pulmonary disease & 11.7 & 12.6 & 10.8 \\
\hline Peripheral artery disease & 3.9 & 2.4 & 5.4 \\
\hline Stroke & 8.9 & 9.4 & 8.5 \\
\hline Gastric ulcer & 21.8 & 22.0 & 21.5 \\
\hline Hand grip strength $(\mathrm{kg})$ & $39.02 \pm 13.76$ & $39.74 \pm 13.67$ & $38.34 \pm 13.86$ \\
\hline SPPB - Total score & $2.088 \pm 0.242$ & $2.089 \pm 0.258$ & $2.086 \pm 0.226$ \\
\hline SPPB - Balance test & $0.976 \pm 0.068$ & $0.981 \pm 0.057$ & $0.971 \pm 0.077$ \\
\hline SPPB — Chair-stand test & $0.484 \pm 0.177$ & $0.483 \pm 0.184$ & $0.485 \pm 0.171$ \\
\hline $\begin{array}{l}\text { SPPB - Walking speed } \\
\text { test }\end{array}$ & $0.611 \pm 0.106$ & $0.608 \pm 0.117$ & $0.613 \pm 0.094$ \\
\hline
\end{tabular}

Values are expressed as mean \pm SD, or percentage. The Short Physical Performance Battery (SPPB) scores range from 0 (worst performance) to 1 (best performance) for each subtask composing it. 


\section{Table 2}

Results from t-test analyses exploring the effects of fosinopril vs. placebo at the end of the six-month follow-up.

\begin{tabular}{lcccc}
\hline & n & $\begin{array}{c}\text { Fosinopril } \\
\text { Mean }(\text { SD })\end{array}$ & $\begin{array}{c}\text { Placebo } \\
\text { Mean }(\text { SD })\end{array}$ & p \\
\hline Hand grip strength (kg) & 225 & $37.044(12.993)$ & $36.898(13.178)$ & 0.57 \\
SPPB - Total score & 222 & $2.113(0.284)$ & $2.096(0.298)$ & 0.23 \\
SPPB - Balance test & 225 & $0.975(0.070)$ & $0.972(0.080)$ & 0.58 \\
SPPB - Chair-stand test & 235 & $0.484(0.201)$ & $0.482(0.196)$ & 0.89 \\
SPPB - Walking speed test & 225 & $0.572(0.120)$ & $0.571(0.114)$ & 0.95 \\
\hline
\end{tabular}

SPPB: Short Physical Performance Battery [ranging from 0 (worst performance) to 1 (best performance) for each subtask composing it]. 